\title{
A Note on Computing the Center of Uncertain Data on the Real Line
}

\author{
Haitao Wang and Jingru Zhang* \\ Department of Computer Science \\ Utah State University, Logan, UT 84322, USA \\ haitao.wang@usu. edu, jingruzhang@aggiemail.usu.edu
}

\begin{abstract}
We consider the one-dimensional one-center problem on uncertain data. We are given a set $\mathcal{P}$ of $n$ (weighted) uncertain points on a real line $L$ and each uncertain point is specified by a probability density function that is a piecewise-uniform function (i.e., a histogram). The goal is to find a point $c$ (the center) on $L$ such that the maximum expected distance from $c$ to all uncertain points of $\mathcal{P}$ is minimized. We present a linear-time algorithm for this problem.
\end{abstract}

Keywords: facility locations, one-center, uncertain data, histogram, lowest point, upper envelope, algorithms, computational geometry

\section{Introduction}

In the real world, data is often associated with uncertainty due to their essence, the measurement inaccuracy, sampling discrepancy, resource limitation, etc [6,9]. In this paper, we consider the one-dimensional one-center problem on uncertain data, defined as follows.

Let $L$ be a real line. Without loss of generality, we assume $L$ is the $x$-axis. Let $\mathcal{P}$ be a set of $n$ uncertain points $\left\{P_{1}, P_{2}, \ldots, P_{n}\right\}$ on $L$, where each uncertain point $P_{i} \in \mathcal{P}$ is specified by its probability density function (pdf) $f_{i}: \mathbb{R} \rightarrow \mathbb{R}^{+} \cup\{0\}$, which is a piecewise-uniform function (i.e., a histogram), consisting of at most $m+1$ pieces (e.g., see Fig. 1). More specifically, for each $P_{i}$, there are $m$ sorted $x$-coordinates $x_{i 1}<x_{i 2}<\ldots<x_{i m}$ and $m-1$ nonnegative values $y_{i 1}, y_{i 2}, \ldots, y_{i, m-1}$ such that $f_{i}(x)=y_{i j}$ for $x_{i j} \leq x<x_{i, j+1}$ with $1 \leq j \leq m-1$. For convenience of discussion, we assume $x_{i 0}=-\infty, x_{i, m+1}=+\infty, y_{i 0}=y_{i m}=0$, and $f_{i}(x)=0$ for $x \in\left(-\infty, x_{i 1}\right) \cup\left[x_{i m},+\infty\right)$.

As discussed in [1], such a histogram function $f_{i}$ can be used to approximate any pdf with arbitrary precision. In particular, for the discrete case where each uncertain point has a finite number of discrete locations, each with a probability, it can also be incorporated by our histogram model using infinitesimal pieces at these locations. Thus, the discrete case is a special case of our histogram model.

With a little abuse of notation, for any (deterministic) point $q$ on $L$, we also use $q$ to denote the $x$ coordinate of $q$. For any uncertain point $P_{i} \in \mathcal{P}$, the expected distance from $q$ to $P_{i}$ is

$$
\operatorname{Ed}\left(q, P_{i}\right)=\int_{-\infty}^{+\infty} f_{i}(x)|x-q| d x
$$

The goal of our one-center problem on $\mathcal{P}$ is to find a (deterministic) point $c^{*}$ on $L$ such that the maximum expected distance from $c^{*}$ to all uncertain points of $\mathcal{P}$ is minimized, and $c^{*}$ is called a center of $\mathcal{P}$.

The algorithm proposed in our previous work [18] can solve the problem in $O(m n \log m n+n \log n \log m n)$ time. In this paper, we present an $O(m n)$ time algorithm. Since the input size of the problem is $\Theta(m n)$, our algorithm runs in linear time, which is optimal.

We should point out that our algorithm is applicable to the weighted case of this problem where each uncertain point $P_{i} \in \mathcal{P}$ has a nonnegative multiplicative weight $w_{i}$ and the weighted expected distance is

\footnotetext{
* Corresponding author.
} 


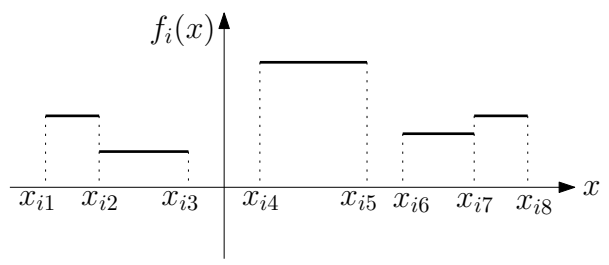

Fig. 1. Illustrating the pdf $f_{i}$ of an uncertain point $P_{i}$ with $m=8$.

considered (i.e., $\left.\operatorname{Ed}\left(q, P_{i}\right)=w_{i} \cdot \int_{-\infty}^{+\infty} f_{i}(x)|x-q| d x\right)$. To solve the weighted case, we can first reduce it to the above unweighted case by changing each value $y_{i j}$ to $w_{i} \cdot y_{i j}$ for every $1 \leq i \leq n$ and $1 \leq j \leq m-1$, and then apply our algorithm for the unweighted case. The running time is still linear. Hence, we will focus our discussion on the unweighted case.

\section{$1.1 \quad$ Related Work}

In our previous work [18], the problem of finding $k$ centers for a set $\mathcal{P}$ of uncertain points on $L$ was studied, and an algorithm of $O(m n \log m n+n \log k \log n \log m n)$ time was proposed. Therefore, when $k=1$, the algorithm runs in $O(m n \log m n+n \log n \log m n)$ time as mentioned above. In addition, the discrete case of the above $k$-center problem (where each uncertain point $P_{i}$ has $m$ discrete locations, each with a probability) was solved in a faster way in $O(m n \log m n+n \log k \log m n)$ time [18], and the discrete one-center problem was solved in $O(m n)$ time [18]. Therefore, our new result in this paper for the one-center problem under the more general histogram model matches the previous result for the discrete case. We also studied the discrete one-center problem for uncertain points on tree networks and proposed a linear-time algorithm in [17].

The deterministic $k$-center problems are classical facility location problems have have been extensively studied. The problem is NP-hard in the plane [13]. Efficient algorithms were known for special cases, e.g., finding the smallest enclosing circle (i.e., the case $k=1$ ) [12], $k$-center on trees $[4,11,14]$. The deterministic $k$-center problem in the one-dimensional space is solvable in $O(n \log n)$ time [5,7,14]. As shown in [18], the deterministic one-center problem in the one-dimensional space can be solved in linear time.

The $k$-center problems on uncertain data in high-dimensional spaces have also been considered. For example, approximation algorithms were given in [8] for different problem models, e.g., the assigned model that is somewhat similar to our problem model and the unassigned model which was relatively easy because it can be reduced to the corresponding deterministic problem [8]. Foul [10] studied the problem of finding the center in the plane to minimize the maximum expected distance from the center to all uncertain points, where each uncertain point has a uniform distribution in a given rectangle. Other facility location problems on uncertain data under various models, e.g., the minmax regret $[2,3,16]$, have also been studied (see [15] for a survey).

\subsection{Our Techniques}

To solve our one-center problem, based on observations, we reduce it to the following geometric problem. Let $\mathcal{H}$ be a set of $n$ unimodal functions in the plane (i.e., when $x$ changes from $-\infty$ to $+\infty$, it first monotonically decreases and then increases) and each function consists of $m$ pieces with each piece being a parabolic arc. We wish to find the lowest point $v^{*}$ in the upper envelope of the functions of $\mathcal{H}$. In the discrete version of the one-center problem, as shown in [18], each parabolic arc of every function of $\mathcal{H}$ is simply a line segment, and thus, $v^{*}$ can be found by applying Megiddo's linear time linear programming algorithm [12]. In our problem, however, since the parabolic arcs of the functions of $\mathcal{H}$ may not be line segments, Megiddo's algorithm in [12] does not work any more. We present a new prune-and-search technique that can compute $v^{*}$ in $O(m n)$ time. This result immediately leads to a linear time algorithm for our one-center problem on $\mathcal{P}$.

Comparing with the linear programming problem, the above geometric problem is more general (i.e., the linear programming problem is a special case of our problem). Our linear time algorithm for the problem may 


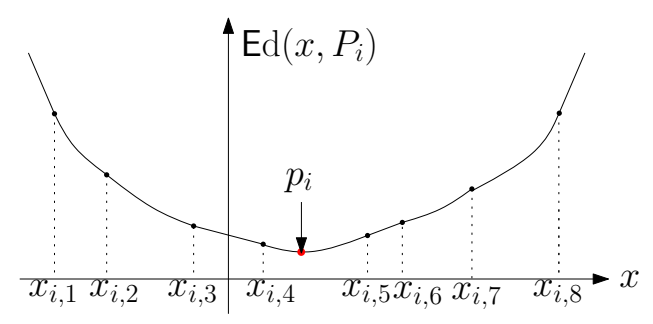

Fig. 2. Illustrating the expected distance function $\operatorname{Ed}\left(x, P_{i}\right)$ for an uncertain point $P_{i}$ with $m=8$; $\operatorname{Ed}\left(x, P_{i}\right)$ is monotonically decreasing for $x \in\left(-\infty, p_{i}\right]$, and increasing for $x \in\left[p_{i},-\infty\right)$.

be interesting in its own right and may find other applications as well. In fact, our result can be extended to more general unimodal functions (see the discussions in Section 4).

\section{Preliminaries}

A function $g: \mathbb{R} \rightarrow \mathbb{R}$ is a unimodal if there exists a value $x^{\prime}$ such that for any $x_{1}<x_{2}, g\left(x_{1}\right) \geq g\left(x_{2}\right)$ holds if $x_{2} \leq x^{\prime}$ and $g\left(x_{1}\right) \leq g\left(x_{2}\right)$ holds if $x^{\prime} \leq x_{1}$, i.e., $g(x)$ is monotonically decreasing on $x \in\left(-\infty, x^{\prime}\right]$ and increasing on $x \in\left[x^{\prime},+\infty\right)$.

Recall that $L$ is the $x$-axis, and for any point $q$ on $L$, we also use $q$ to denote the $x$-coordinate of $q$. Therefore, the values of $\mathbb{R}$ correspond to the points of $L$. In the following, we will use "the values of $\mathbb{R}$ " and "the points of $L$ " interchangeably.

Consider any uncertain point $P_{i}$ of $\mathcal{P}$. If we consider the expected distance $\operatorname{Ed}\left(x, P_{i}\right)$ as a function of the points $x$ on $L($ or $x \in \mathbb{R})$, the following observation has been proved in [18]. Note that the $m$ coordinates $x_{i 1}, \ldots, x_{i m}$ of $P_{i}$ are already given sorted.

Lemma 1. [18] The function $\mathrm{Ed}\left(x, P_{i}\right)$ for $x \in \mathbb{R}$ is unimodal. More specifically, there exists a point $p_{i} \in L$ such that $\operatorname{Ed}\left(x, P_{i}\right)$ is monotonically decreasing on $x \in\left(-\infty, p_{i}\right]$ and increasing on $x \in\left[p_{i},+\infty\right)$ (e.g., see Fig. 2). In addition, $\operatorname{Ed}\left(x, P_{i}\right)$ is a parabolic arc (of constant complexity) on the interval $\left[x_{i k}, x_{i, k+1}\right)$ for each $0 \leq k \leq m$, and can be explicitly computed in $O(m)$ time.

The point $p_{i}$ in Lemma 1 is referred to as a centroid of $P_{i}$ and can be easily computed in $O(m)$ time after $\operatorname{Ed}\left(x, P_{i}\right)$ is explicitly computed in $O(m)$ time [18]. In fact, a point $q \in L$ is a centroid of $P_{i}$ if and only if $\int_{-\infty}^{q} f_{i}(x)=0.5$ and $\int_{q}^{+\infty} f_{i}(x)=0.5$. Note that the centroid of $P_{i}$ may not be unique. This case happens when there exists an interval on the $x$-axis such that for any point $q$ in this interval both $\int_{-\infty}^{q} f_{i}(x)=0.5$ and $\int_{q}^{+\infty} f_{i}(x)=0.5$ hold, and thus $\operatorname{Ed}\left(x, P_{i}\right)$ is a constant when $x$ is in this interval and any point $q$ in this interval is a centroid. If the centroid of $P_{i}$ is not unique, then we use $p_{i}$ to refer to an arbitrary such centroid. For a similar reason, the center of $\mathcal{P}$ may also not be unique, in which case our algorithm will find one such center.

The following corollary can be easily obtained based on Lemma 1 and binary search on the sorted list of $x_{i 1}, x_{i 2}, \ldots, x_{i m}$.

Corollary 1. [18] For each uncertain point $P_{i}$, with $O(m)$ time preprocessing, the value $\operatorname{Ed}\left(x^{\prime}, P_{i}\right)$ for any query value $x^{\prime}$ can be computed in $O(\log m)$ time.

Let $\mathcal{H}$ denote the set of all functions $\operatorname{Ed}\left(x, P_{i}\right)$ for $i=1,2, \ldots, n$. Since the center $c^{*}$ of $\mathcal{P}$ is a point on $L$ that minimizes the value $\max _{i=1}^{n} \operatorname{Ed}\left(x, P_{i}\right)$, i.e., $c^{*}=\operatorname{argmin}_{x \in L} \max _{i=1}^{n} \operatorname{Ed}\left(x, P_{i}\right)$, $c^{*}$ is equal to the $x$-coordinate of the lowest point $v^{*}$ in the upper envelope of $\mathcal{H}$. Therefore, to compute $c^{*}$, it is sufficient to find $v^{*}$.

As shown in [18] and mentioned in Section 1.2, in the discrete case where each uncertain point $P_{i}$ has $m$ discrete locations (each with a probability), each function $\operatorname{Ed}\left(x, P_{i}\right)$ consists of $m+1$ pieces with each piece 
being a special parabolic arc: a line segment. Consequently, to compute $v^{*}$ is equivalent to computing the lowest point in the upper envelope of the $n(m+1)$ extending lines of all line segments of all functions of $\mathcal{H}$, which can be done in $O(m n)$ time by Megiddo's linear time linear programming algorithm [12].

In our problem, since each piece of every function of $\mathcal{H}$ may be a general parabolic arc, Megiddo's algorithm [12], which heavily relies on the properties of lines, does not work any more. In the next section, we present a new prune-and-search algorithm that can compute $v^{*}$ in $O(m n)$ time, and it can be considered as an extension of Megiddo's algorithm [12].

\section{Compute the Lowest Point $v^{*}$ in the Upper Envelope of $\mathcal{H}$}

In this section, we present an algorithm that can compute $v^{*}$ in $O(m n)$ time. Note that the lowest point of the upper envelope of $\mathcal{H}$ may not be unique, in which case we use $v^{*}$ to represent an arbitrary such lowest point. We assume the preprocessing in Corollary 1 has been done for every uncertain point $P_{i} \in \mathcal{P}$, which takes $O(m n)$ time in total. Denote by $x^{*}$ the $x$-coordinate of $v^{*}$. We first present an $O(n \log m)$ time algorithm for solving the following decision problem: given any value $x^{\prime}$, determine whether $x^{\prime}<x^{*}, x^{\prime}=x^{*}$, or $x^{\prime}>x^{*}$. Let $\mathcal{U}$ denote the upper envelope of $\mathcal{H}$.

\subsection{A Decision Algorithm}

Consider any value $x^{\prime}$. To solve the decision problem, the main idea is to compute the intersection of $\mathcal{U}$ and the vertical line $x=x^{\prime}$, and then determine whether $x^{\prime}<x^{*}, x^{\prime}=x^{*}$, or $x^{\prime}>x^{*}$ based on the local information of $\mathcal{U}$ at the above intersection. The details are given below. Denote by $l\left(x^{\prime}\right)$ the vertical line $x=x^{\prime}$.

For any uncertain point $P_{i} \in \mathcal{P}$, by Corollary 1 , we can compute the intersection of the function $\operatorname{Ed}\left(x, P_{i}\right)$ and $l\left(x^{\prime}\right)$ in $O(\log m)$ time, and let $q_{i}$ denote the above intersection. In fact, the parabolic $\operatorname{arc}$ of $\operatorname{Ed}\left(x, P_{i}\right)$ that contains $q_{i}$ can also be determined. We let $S_{i}$ denote the above parabolic arc. In the case that $q_{i}$ is the common endpoint of two parabolic arcs, we let $S_{i}$ denote the set of both parabolic arcs. Hence $\left|S_{i}\right| \leq 2$ holds in either case. In summary, $q_{i}$ and $S_{i}$ can be determined in $O(\log m)$ time.

We compute $q_{i}$ and $S_{i}$ for every uncertain point $P_{i} \in \mathcal{P}$, which takes $O(n \log m)$ time in total with the preprocessing in Corollary 1. Let $Q=\left\{q_{i} \mid 1 \leq i \leq n\right\}$. Next, we find the highest point $q\left(x^{\prime}\right)$ of $Q$, and clearly, $q\left(x^{\prime}\right)$ is the intersection of $l\left(x^{\prime}\right)$ and the upper envelope $\mathcal{U}$. In case $q\left(x^{\prime}\right)$ is equal to multiple points of $Q$, we let $I\left(x^{\prime}\right)$ denote the index set such that for each $i \in I\left(x^{\prime}\right), q_{i}=q\left(x^{\prime}\right)$. Let $S\left(x^{\prime}\right)=\cup_{i \in I\left(x^{\prime}\right)} S_{i}$. Note that after $q_{i}$ and $S_{i}$ for every $P_{i} \in \mathcal{P}$ are computed, we can obtain $q\left(x^{\prime}\right), I\left(x^{\prime}\right)$ and $S\left(x^{\prime}\right)$ in $O(n)$ time.

Based on $q\left(x^{\prime}\right)$ and the set $S\left(x^{\prime}\right)$ of parabolic arcs, it is not difficult to see that we can solve the decision problem in the following way. If all parabolic arcs of $S\left(x^{\prime}\right)$ are strictly decreasing at $q\left(x^{\prime}\right)$, then $x^{\prime}<x^{*}$. If all parabolic arcs of $S\left(x^{\prime}\right)$ are strictly increasing at $q\left(x^{\prime}\right)$, then $x^{\prime}>x^{*}$. Otherwise, $x^{\prime}=x^{*}$. Clearly, with $q\left(x^{\prime}\right)$ and $S\left(x^{\prime}\right)$, the above can be determined in $O(n)$ time.

Therefore, we obtain the following result.

Lemma 2. With $O(m n)$ time preprocessing, given any value $x^{\prime}$, we can determine whether $x^{\prime}<x^{*}, x^{\prime}=x^{*}$, or $x^{\prime}>x^{*}$, in $O(n \log m)$ time.

\subsection{Observations}

We first give the following lemma, which will help us to prune uncertain points of $\mathcal{P}$ in our prune-and-search algorithm.

Lemma 3. For any two uncertain points $P_{i}$ and $P_{j}$, the upper envelope of $\operatorname{Ed}\left(x, P_{i}\right)$ and $\operatorname{Ed}\left(x, P_{j}\right)$ has $O(m)$ complexity and can be computed in $O(m)$ time. 
Proof. Consider two uncertain points $P_{i}$ and $P_{j}$. We first analyze the intersections of the functions $\operatorname{Ed}\left(x, P_{i}\right)$ and $\operatorname{Ed}\left(x, P_{j}\right)$. Recall that each of these two functions has $m+1$ parabolic arcs.

We use $\operatorname{Ed}_{L}\left(x, P_{i}\right)$ to denote the part of $\operatorname{Ed}\left(x, P_{i}\right)$ to the left of the centroid $p_{i}$ (i.e., $x \in\left(-\infty, p_{i}\right]$ ), and use $\operatorname{Ed}_{R}\left(x, P_{i}\right)$ to denote the part of $\operatorname{Ed}\left(x, P_{i}\right)$ to the right of $p_{i}$ (i.e., $x \in\left[p_{i},+\infty\right)$ ). Hence, $\operatorname{Ed}_{L}\left(x, P_{i}\right)$ is monotonically decreasing and $\operatorname{Ed}_{R}\left(x, P_{i}\right)$ is monotonically increasing. We define $\operatorname{Ed}_{L}\left(x, P_{j}\right)$ and $\operatorname{Ed}_{R}\left(x, P_{j}\right)$ for $P_{j}$ similarly.

Since $\operatorname{Ed}_{L}\left(x, P_{i}\right)$ is monotonically increasing and $\operatorname{Ed}_{R}\left(x, P_{j}\right)$ is monotonically decreasing, $\operatorname{Ed}_{L}\left(x, P_{i}\right)$ can intersect $\operatorname{Ed}_{R}\left(x, P_{j}\right)$ transversally at most once. Similarly, $\operatorname{Ed}_{R}\left(x, P_{i}\right)$ can intersect $\operatorname{Ed}_{L}\left(x, P_{j}\right)$ transversally at most once.

Consider $\operatorname{Ed}_{L}\left(x, P_{i}\right)$ and $\operatorname{Ed}_{L}\left(x, P_{j}\right)$. Since both of them are monotonically decreasing, a parabolic arc of $\operatorname{Ed}_{L}\left(x, P_{i}\right)$ can intersect any parabolic arc of $\operatorname{Ed}_{L}\left(x, P_{j}\right)$ transversally at most twice (this is because both parabolic arcs are decreasing). Therefore, $\operatorname{Ed}_{L}\left(x, P_{i}\right)$ can intersect $\operatorname{Ed}_{L}\left(x, P_{j}\right)$ transversally $O(m)$ times. Similarly, $\operatorname{Ed}_{R}\left(x, P_{i}\right)$ can intersect $\operatorname{Ed}_{R}\left(x, P_{j}\right)$ transversally $O(m)$ times.

The above discussion leads to the conclusion that the two functions $\operatorname{Ed}\left(x, P_{i}\right)$ and $\operatorname{Ed}\left(x, P_{j}\right)$ can intersect each other transversally at most $O(m)$ times. This implies that the upper envelope of $\operatorname{Ed}\left(x, P_{i}\right)$ and $\operatorname{Ed}\left(x, P_{j}\right)$ is of complexity $O(m)$. Since both $\operatorname{Ed}\left(x, P_{i}\right)$ and $\operatorname{Ed}\left(x, P_{j}\right)$ can be computed in $O(m)$ time by Lemma 1, their upper envelope can be computed by a simple sweeping algorithm in $O(m)$ time. The lemma thus follows.

Lemma 3 implies the following corollary.

Corollary 2. For any two uncertain points $P_{i}$ and $P_{j}$, in $O(m)$ time we can compute a sorted list of values $-\infty=x_{0}<x_{1}<x_{2}<\ldots<x_{t}<x_{t+1}=+\infty$ with $t=O(m)$ such that either $\operatorname{Ed}\left(x, P_{i}\right) \leq \operatorname{Ed}\left(x, P_{j}\right)$ or $\operatorname{Ed}\left(x, P_{i}\right)>\operatorname{Ed}\left(x, P_{j}\right)$ holds when $x \in\left[x_{k}, x_{k+1}\right]$ for any $0 \leq k \leq t$.

Recall that $x^{*}$ is the $x$-coordinate of $v^{*}$. Corollary 2 implies that if we know $x^{*}$ is contained in some interval $\left[x_{k}, x_{k+1}\right]$ as defined in Corollary 2, then at least one of $P_{i}$ and $P_{j}$ can be pruned, and more specifically, if $\operatorname{Ed}\left(x, P_{i}\right) \leq \operatorname{Ed}\left(x, P_{j}\right)$ when $x \in\left[x_{k}, x_{k+1}\right]$, then for computing $v^{*}, P_{i}$ can be discarded. In the following, let $X(i, j)$ denote the set of sorted $x$-coordinates as defined in Corollary 2. Thus, it holds that $|X(i, j)| \leq C m$ for some constant $C$ for any two uncertain points $P_{i}$ and $P_{j}$ of $\mathcal{P}$.

\subsection{Computing the Lowest Point $v^{*}$}

In the sequel, we present our algorithm for computing $v^{*}$ based on the prune-and-search techniques. Below, we show that our algorithm can prune $n / 4$ uncertain points of $\mathcal{P}$ in $O(m n)$ time.

We arbitrarily assign the uncertain points of $\mathcal{P}$ into $n / 2$ pairs. Let $\Sigma$ denote all such pairs. For each pair $\left(P_{i}, P_{j}\right) \in \Sigma$, we compute $X(i, j)$ and the upper envelope of $\operatorname{Ed}\left(x, P_{i}\right)$ and $\operatorname{Ed}\left(x, P_{j}\right)$ in $O(m)$ time by Lemma 3. Let $\mathcal{X}=\bigcup_{\left(P_{i}, P_{j}\right) \in \Sigma} X(i, j)$. Since $\Sigma$ has $n / 2$ pairs, $\mathcal{X}$ can be computed in $O(m n)$ time by Lemma 3 and $|\mathcal{X}| \leq C m n / 2$.

We find the median $x_{m}$ of $\mathcal{X}$ in $O(m n)$ time. By using our decision algorithm in Lemma 2, we can determine whether $x_{m}<x^{*}, x_{m}=x^{*}$, or $x_{m}>x^{*}$ in $O(n \log m)$ time. If $x_{m}=x^{*}$, then $x_{m}$ is the center of $\mathcal{P}$ and we are done with the algorithm. Otherwise, if $x_{m}<x^{*}$, then we eliminate all values in $\mathcal{X}$ that are smaller than or equal to $x_{m}$; if $x_{m}>x^{*}$, then we eliminate all values in $\mathcal{X}$ that are larger than or equal to $x_{m}$. In either case, no more than $|\mathcal{X}| / 2$ values of $\mathcal{X}$ will remain. Next, we continue the above procedure recursively on the remaining values of $\mathcal{X}$ until the number of remaining values is no more than $n / 4$. Since initially $|\mathcal{X}| \leq C m n / 2$, the number of recursive steps is bounded by $O(\log (2 C m))=O(\log m+\log C+1)$.

We claim that the total running time of the above recursive procedure is $O(m n)$. Indeed, the total time for finding the medians in all recursive steps is bounded by $O(|\mathcal{X}|+|\mathcal{X}| / 2+|\mathcal{X}| / 4+\cdots)=O(|\mathcal{X}|)=O(m n)$. In each recursive step, we need to call the decision algorithm once, and therefore, the total time of the decision algorithm in all recursive steps is $O\left(n \log ^{2} m\right)$. Hence, the above claim is proved.

We should point out that one may want to keep doing the above recursive procedure until $X$ has at most one or two remaining values, which would need $\Theta(\log m n)$ recursive steps; but according to our above time complexity analysis, it would take $O(m n+n \log m \log m n)$ time, which may not necessarily be bounded by 
$O(m n)$ (e.g., when $m=o(\log n))$. In fact, performing the above recursive steps until the remaining values of $\mathcal{X}$ is at most $n / 4$ is an interesting and crucial part of our techniques.

Let $\mathcal{X}^{\prime}$ be the set of remaining values of $\mathcal{X}$. Hence, $\left|\mathcal{X}^{\prime}\right| \leq n / 4$. Let $x^{\prime}$ and $x^{\prime \prime}$ denote the smallest and largest values of $\mathcal{X}^{\prime}$, respectively. According to our above way of computing $\mathcal{X}^{\prime}, \mathcal{X}^{\prime}$ consists of all values of $\mathcal{X}$ in $\left[x^{\prime}, x^{\prime \prime}\right]$, and further, $x^{*} \in\left[x^{\prime}, x^{\prime \prime}\right]$. Since $\left|\mathcal{X}^{\prime}\right| \leq n / 4$, there are at most $n / 4$ pairs $\left(P_{i}, P_{j}\right)$ of $\Sigma$ such that $X(i, j)$ contains a value in $\mathcal{X}^{\prime}$. In other words, we have the following observation.

Observation 1 There are at least $n / 4$ pairs $\left(P_{i}, P_{j}\right)$ of $\Sigma$ such that $X(i, j)$ does not contain any value of $\mathcal{X}^{\prime}$ (i.e., no value of $X(i, j)$ is in the interval $\left[x^{\prime}, x^{\prime \prime}\right]$ ).

Let $\Sigma^{\prime}$ denote the set of pairs of $\Sigma$ that satisfy the condition in Observation 1 (i.e., for each pair $\left(P_{i}, P_{j}\right)$ of $\Sigma^{\prime}$, no value of $X(i, j)$ is in the interval $\left.\left[x^{\prime}, x^{\prime \prime}\right]\right)$. Hence, $\left|\Sigma^{\prime}\right| \geq n / 4$. Consider any pair $\left(P_{i}, P_{j}\right) \in \Sigma^{\prime}$. Let $X(i, j)=x_{1}, x_{2}, \ldots, x_{t}$ with $x_{0}=-\infty$ and $x_{t+1}=+\infty$. By Observation 1 , since no value of $X(i, j)$ is in the interval $\left[x^{\prime}, x^{\prime \prime}\right],\left[x^{\prime}, x^{\prime \prime}\right]$ is contained in $\left[x_{k}, x_{k+1}\right]$ for some $k$ with $0 \leq k \leq t$. Thus, either $\operatorname{Ed}\left(x, P_{i}\right) \leq \operatorname{Ed}\left(x, P_{j}\right)$ or $\operatorname{Ed}\left(x, P_{i}\right)>\operatorname{Ed}\left(x, P_{j}\right)$ holds for $x \in\left[x^{\prime}, x^{\prime \prime}\right]$. Without loss of generality, we assume $\operatorname{Ed}\left(x, P_{i}\right) \leq \operatorname{Ed}\left(x, P_{j}\right)$ holds for $x \in\left[x^{\prime}, x^{\prime \prime}\right]$. Since $x^{*} \in\left[x^{\prime}, x^{\prime \prime}\right]$, we obtain that $\operatorname{Ed}\left(x^{*}, P_{i}\right) \leq \operatorname{Ed}\left(x^{*}, P_{j}\right)$. Therefore, $P_{i}$ can be discarded. Since $\left|\Sigma^{\prime}\right| \geq n / 4$, we can discard at least $n / 4$ uncertain points of $\mathcal{P}$.

Recall that $\mathcal{X}^{\prime}$ has been computed and $\left|\mathcal{X}^{\prime}\right| \leq n / 4$. The above pruning procedure can be done in $O(m n)$ time. Indeed, we can find $x^{\prime}$ and $x^{\prime \prime}$ in $\mathcal{X}^{\prime}$ in $O(n)$ time. To determine the set $\Sigma^{\prime}$, suppose for each element $x \in \mathcal{X}$, we have associated the pair $\left(P_{i}, P_{j}\right)$ with $x$ such that $x \in X(i, j)$; then $\Sigma^{\prime}$ can be determined in $O(n)$ time by scanning $\mathcal{X}^{\prime}$. Next, for each pair $\left(P_{i}, P_{j}\right)$ of $\Sigma^{\prime}$, we determine the interval $\left[x_{k}, x_{k+1}\right]$ of $X_{i j}$ (as defined above) that contains $\left[x^{\prime}, x^{\prime \prime}\right]$, which can be easily done in $O(m)$ since the values of $X(i, j)$ have already been sorted and $|X(i, j)|=O(m)$ by Corollary 2. Since the upper envelope of $P_{i}$ and $P_{j}$ has been computed, we can also determine whether $\operatorname{Ed}\left(x, P_{i}\right) \leq \operatorname{Ed}\left(x, P_{j}\right)$ or $\operatorname{Ed}\left(x, P_{i}\right)>\operatorname{Ed}\left(x, P_{j}\right)$ holds for $x \in\left[x^{\prime}, x^{\prime \prime}\right]$ in $O(m)$ time. Consequently, we can prune one of $P_{i}$ and $P_{j}$. Hence, the pruning procedure can be done in $O(m n)$ time.

The above discussion shows that within $O(m n)$ time, we can prune at least $n / 4$ uncertain points of $\mathcal{P}$ such that the center $c^{*}$ is determined only by the remaining at most $3 n / 4$ uncertain points of $\mathcal{P}$.

We continue the above procedure recursively on the remaining uncertain points of $\mathcal{P}$ until a constant number of uncertain points of $\mathcal{P}$ remain. The total running time satisfies the recurrence $T(m, n)=$ $T(m, 3 n / 4)+O(m n)$. Solving the recurrence yields $T(m, n)=O(m n)$. Let $\mathcal{P}^{\prime}$ denote the set of remaining uncertain points of $\mathcal{P}$. We have $\left|\mathcal{P}^{\prime}\right|=O(1)$. Let $\mathcal{H}^{\prime}$ denote the set of functions $\operatorname{Ed}\left(x, P_{i}\right)$ for all $P_{i} \in \mathcal{P}^{\prime}$, and let $\mathcal{U}^{\prime}$ be the upper envelope of the functions of $\mathcal{H}^{\prime}$. According to our above pruning algorithm, $v^{*}$ is also the lowest point of $\mathcal{U}^{\prime}$. Note that in the case that the lowest point of $\mathcal{U}^{\prime}$ is not unique, our pruning algorithm above makes sure that every lowest point of $\mathcal{U}^{\prime}$ is a lowest point of $\mathcal{U}$ (we omit the detailed discussions).

Finally, we find the lowest point of $\mathcal{U}^{\prime}$ by constructing it explicitly, which can be done in $O(m)$ time by a sweeping algorithm, as follows. We sweep a vertical line $l$ from left to right. During the sweeping, we maintain the intersections of $l$ with all functions of $\mathcal{H}^{\prime}$ in the order sorted by their $y$-coordinates (note that the number of such intersections is $O(1)$ since $\left|\mathcal{H}^{\prime}\right|=O(1)$ ). An event happens when $l$ hits a vertex of some function or an intersection of two functions. Clearly, all functions of $\mathcal{H}^{\prime}$ have $O(m)$ vertices since $\left|\mathcal{H}^{\prime}\right|=O(1)$. As discussed in the proof of Lemma 3, every two functions of $\mathcal{H}^{\prime}$ can intersect (transversally) $O(m)$ times. Therefore, the number of (transversal) intersections among all functions of $\mathcal{H}^{\prime}$ is $O(m)$ since $\left|\mathcal{H}^{\prime}\right|=O(1)$. Hence, the total number of events is $O(m)$. Processing each event can be done in constant time since $\left|\mathcal{H}^{\prime}\right|=O(1)$. Therefore, we can explicitly construct $\mathcal{U}^{\prime}$ and thus find its lowest point in $O(m)$ time.

Combining all above efforts, the lowest vertex $v^{*}$ of $\mathcal{H}$ can be computed in $O(m n)$ time. Thus, the center of $\mathcal{P}$ can be computed in $O(m n)$ time.

Theorem 1. The center of $\mathcal{P}$ can be computed in $O(m n)$ time.

\section{Concluding Remarks}

In fact, we can easily extend our algorithm for the above geometric problem to more general functions. Specifically, let $\mathcal{H}$ be a set of $n$ unimodal functions in the plane such that each function consists of $m$ pieces 
with each piece being a constant-sized algebraic curve segment with the following property: any two such curve segments of all functions of $\mathcal{H}$ intersect (transversally) at most a constant number of times. Then, our techniques can be used to find the lowest point in the upper envelope of all functions of $\mathcal{H}$ in $O(m n)$ time.

It would be interesting to see whether the techniques proposed in this paper can be used to solve other related problems.

\section{Acknowledgment}

This research was supported in part by NSF under Grant CCF-1317143.

\section{References}

1. P.K. Agarwal, S.-W. Cheng, Y. Tao, and K. Yi. Indexing uncertain data. In Proc. of the 28th Symposium on Principles of Database Systems (PODS), pages 137-146, 2009.

2. I. Averbakh and S. Bereg. Facility location problems with uncertainty on the plane. Discrete Optimization, $2: 3-34,2005$.

3. I. Averbakh and O. Berman. Minimax regret p-center location on a network with demand uncertainty. Location Science, 5:247-254, 1997.

4. R. Chandrasekaran and A. Tamir. Polynomially bounded algorithms for locating $p$-centers on a tree. Mathematical Programming, 22(1):304-315, 1982.

5. D.Z. Chen, J. Li, and H. Wang. Efficient algorithms for one-dimensional $k$-center problems. Theoretical Computer Science, 592:135-142, 2015.

6. R. Cheng, J. Chen, and X. Xie. Cleaning uncertain data with quality guarantees. Proceedings of the VLDB Endowment, 1(1):722-735, 2008.

7. R. Cole. Slowing down sorting networks to obtain faster sorting algorithms. Journal of the ACM, 34(1):200-208, 1987.

8. G. Cormode and A. McGregor. Approximation algorithms for clustering uncertain data. In Proc. of the 27t Symposium on Principles of Database Systems (PODS), pages 191-200, 2008.

9. X. Dong, A.Y. Halevy, and C. Yu. Data integration with uncertainty. In Proceedings of the 33rd International Conference on Very Large Data Bases, pages 687-698, 2007.

10. A. Foul. A 1-center problem on the plane with uniformly distributed demand points. Operations Research Letters, 34(3):264-268, 2006.

11. G.N. Frederickson. Parametric search and locating supply centers in trees. In Proc. of the 2nd International Workshop on Algorithms and Data Structures (WADS), pages 299-319, 1991.

12. N. Megiddo. Linear-time algorithms for linear programming in $R^{3}$ and related problems. SIAM Journal on Computing, 12(4):759-776, 1983.

13. N. Megiddo and K.J. Supowit. On the complexity of some common geometric location problems. SIAM Journal on Comuting, 13:182-196, 1984.

14. N. Megiddo and A. Tamir. New results on the complexity of p-centre problems. SIAM Journal on Computing, 12(4):751-758, 1983.

15. L.V. Snyder. Facility location under uncertainty: a review. IIE Transactions, 38:537-554, 2006.

16. H. Wang. Minmax regret 1-facility location on uncertain path networks. European Journal of Operational Research, 239:636-643, 2014.

17. H. Wang and J. Zhang. Computing the center of uncertain points on tree networks. In Proc. of the 14th Algorithms and Data Structures Symposium (WADS), pages 606-618, 2015.

18. H. Wang and J. Zhang. One-dimensional k-center on uncertain data. Theoretical Computer Science, 602:114-124, 2015 . 